\title{
Ética, Desarrollo Sostenible y Responsabilidad Social desde la docencia en instituciones de educación superior latinoamericanas
}

\author{
Ethics, Sustainable Development and Social Responsability from university teaching in \\ Latin American higher education institutions
}

Fabiola García Rangel $\bowtie$

Universidad Michoacana de San Nicolás de Hidalgo

fabiola.garcia@umich.mx

Morelia Michoacán, México

\section{Rosa Vega Cano}

Universidad Michoacana de San Nicolás de Hidalgo rosa.vega@umich.mx

Morelia Michoacán, México

\section{François Vallaeys}

Universidad del Pacífico

f.vallaeys@up.edu.pe

Lima, Perú

\begin{abstract}
Resumen:
El presente documento muestra los resultados de un análisis de las reflexiones vertidas por docentes y funcionarios universitarios de ocho países de América Latina, quienes participaron en las dos ediciones de un diplomado internacional, producto de la colaboración entre la Unión de Responsabilidad Social Universitaria Latinoamericana (URSULA) y la Universidad Michoacana de San Nicolás de Hidalgo, cuyo eje de trabajo fue el análisis del impacto que tiene la labor docente sobre el desarrollo sostenible.

El estudio se desarrolló desde un enfoque mixto. Mediante un análisis de contenido se analizaron cualitativamente 410 contribuciones realizadas por quienes participaron en el diplomado y, posteriormente, se llevó a cabo un análisis de frecuencias para cuantificarlas en torno a los ámbitos y metas del Modelo de Responsabilidad Social Universitaria propuesto por URSULA.

El trabajo concluye señalando que la docencia universitaria debe ser revalorada, en términos de su contribución al desarrollo sostenible, pues a través de su ejercicio se materializa la pertinencia social de la formación profesional mediante el desarrollo de prácticas y contenidos que acerquen al estudiantado a la realidad económica, social y ambiental en que viven.
\end{abstract}

Palabras clave: Docencia universitaria; Ética; Desarrollo Sostenible; Responsabilidad Social; América Latina.

$\bowtie$ Autor por correspondencia

Cómo referenciar:

García Rangel, F., Vega Cano, R., \& Vallaeys, F. (2022).

Ética, Desarrollo Sostenible y Responsabilidad Social desde la docencia en instituciones de educación superior latinoamericanas.

Emerging Trends in Education, (4)8A, 48-61

https://doi.org/10.19136/etie.a4n8A.4729

Disponible en:

https://revistas.ujat.mx/index.php/emerging

DOI:

https://doi.org/10.19136/etie.a4n8A.4729

Recibido:

Aceptado:

18/09/2021

$14 / 12 / 2021$

Publicado:

05/01/2022

Emerging Trends in Education

e ISSN: $2594-2840$

Volumen 4, Número 8A, Enero - Junio 2022

\begin{abstract}
:
This document shows the results of an analysis of the reflections expressed by university professors and authorities from eight Latin American countries who participated in the two editions of an international diploma course. This course is a product of the collaboration between the Union of Latin American University Social Responsibility and the Universidad Michoacana de San Nicolás de Hidalgo, whose main focus was the analysis of the impact of teaching on sustainable development.

The study was developed from a mixed-method approach. Through a content analysis, more than 410 contributions made by those who participated in the diploma course were qualitatively analyzed and, subsequently, a frequency analysis was carried out to quantify them in terms of the areas and goals of the University Social Responsibility Model proposed by URSULA.

The paper concludes by pointing out that university teaching should be revalued in terms of its contribution to sustainable development, since through its exercise, the social relevance of professional training is materialized through the development of practices and contents that bring students closer to the economic, social and environmental reality in which they live.
\end{abstract}

Keywords: University teaching; Ethics; Sustainable Development; Social Responsibility; Latin America. 


\section{Ética, Desarrollo Sostenible y Responsabilidad Social desde la docencia en instituciones de educación superior latinoamericanas}

\section{| Introducción}

La Responsabilidad Social Universitaria (RSU) ha planteado una redefinición del rol de la universidad, desde principios del siglo XXI, destacando que la incidencia que puede tener en el territorio va más allá de las actividades de extensión extra-académicas que se llevan a cabo fuera de la institución y aparte de su quehacer diario de formación e investigación; la influencia que ejercen las Instituciones de Educación Superior (IES) está implícita en todos los procesos internos relacionados con el cumplimiento de sus funciones sustantivas: administración del campus, formación profesional y ciudadana, investigación y difusión de conocimientos, participación institucional en las agendas de desarrollo locales, nacionales e internacionales. (Vallaeys \& Alvarez Rodríguez, 2019).

En analogía al modelo heliocéntrico de Nicolás Copérnico para el estudio de los planetas y el universo, Vallaeys (2020a, pp. 114-115) propone una revolución copernicana para llamar la atención en la necesidad de "pasar de los actos a los impactos", explicando que

[...] cuando consideramos a la educación superior desde fuera, desde los problemas sociales y ambientales y sus demandas de solución, la mirada descubre en qué medida las rutinas de las IES contribuyen y refuerzan sin querer a los problemas sociales crónicos, en qué medida desaprovechan sus procesos internos para hacer cosas que no le sirven a la sociedad [...], en qué medida los generosos actos de extensión solidaria influyen muy poco y en qué medida las orientaciones de gestión administrativa y académica [...] van muchas veces en contra de las buenas declaraciones de intención de los discursos misionales.

Este cambio de paradigma en el abordaje de la misión social de la educación superior, tiene importantes consecuencias en la labor docente, puesto que obliga a cuestionar cuáles son los impactos (positivos y negativos) del proceso de enseñanza-aprendizaje en la sociedad y el medioambiente; parte de exigir la reflexión en torno a preguntas como las siguientes: ¿la formación que se imparte en la universidad es socialmente responsable ante los posibles riesgos de consecuencias negativas del desempeño profesional de los futuros egresados, en términos de desarrollo humano y sostenibilidad ambiental? ¿El estudiantado se forma con plena consciencia de los desafíos locales y globales del desarrollo sostenible relacionados con su carrera profesional? ¿La universidad cumple con su deber de innovación social para ayudar en la solución de los problemas del entorno desde el ejercicio de sus funciones sustantivas de formación e investigación? Estas preguntas, éticas y disciplinarias a la vez, reclaman revisar el currículo, los métodos pedagógicos, los perfiles y los criterios de evaluación de la tarea docente, porque ya no se trata de agregar a la formación, mediante un "servicio social" por ejemplo, una dimensión de solidaridad, sino de asegurar que la formación misma sea solidaria, y no vaya de modo oculto en contra de los fines del desarrollo humano 
Ética, Desarrollo Sostenible y Responsabilidad Social desde la docencia en instituciones de educación superior latinoamericanas.

sostenible.

Esta visión de la RSU es coherente con las propuestas emanadas de la discusión de los países en torno a las estrategias que consideran necesarias para atender los grandes temas del desarrollo, con los compromisos que han asumido al suscribir la Agenda 2030 y sus 17 Objetivos de Desarrollo Sostenible (ODS) y con lo cual se confirma la visión de la educación como un motor de desarrollo.

En palabras de Ramos (2021, p. 91),

[...] la educación tiene un papel crucial para el logro de los ODS, dada su labor de generación y difusión del conocimiento y su preeminente situación dentro de la sociedady, más aún cuando se considera que todos los ODS basan sus argumentos en el conocimiento, solicitan nuevos conocimientos y sugieren cambios futuros con referencia a la creencia en la ciencia [...].

Ante este contexto, existen nuevas demandas para los docentes, ya que, al ser interpeladas por la agenda del desarrollo sostenible, las IES requieren de profesores que reconozcan la relevancia de su labor en la formación de profesionales comprometidos y responsables de la sostenibilidad del planeta, por lo que es urgente brindarles la formación y el apoyo que requieren para hacer frente a tal desafío (Global Alliance of Tertiary and Higher Education Sustainability and Student Networks [EAUC \& ACTS], 2021).

De ahí la necesidad de constituir una capacitación específicamente dirigida al personal docente y el liderazgo académico de las instituciones de educación superior latinoamericanas, destinada a articular docencia con RSU, que permita una reflexión crítica entre colegas de diversos países del continente.

La Unión de Responsabilidad Social Universitaria Latinoamericana (URSULA) ha sido un espacio pertinente para dicho propósito. Desde 2016 ha asumido el compromiso de "promover una inteligencia colectiva y colaborativa a cerca del rol social de las Instituciones de Educación Superior en tiempos de riesgos globales y urgencias de cambios." (Unión de Responsabilidad Social Universitaria Latinoamericana [URSULA], 2020).

Por su parte, la Universidad Michoacana de San Nicolás de Hidalgo (UMSNH) -Universidad Michoacana, en adelante- ha emprendido acciones relevantes al respecto; de fines de 2012 a principios de 2013, realizó el primer ejercicio de capacitación docente con el Curso-Taller Formación de Actores de Responsabilidad Social Universitaria. Este ejercicio se llevó a cabo con la guía y acompañamiento del actual presidente de URSULA y se ha convertido en un importante antecedente en programas de actualización docente en los temas de ética, responsabilidad social y desarrollo sostenible.

La trayectoria y experiencia lograda por esta Universidad del centro-occidente de México en la formación docente a través de cursos semi-presenciales y virtuales en torno al tema de la RSU, y el interés de URSULA por revalorar el rol docente en la implementación de este enfoque en la educación superior, se unieron para elaborar una propuesta formativa en la que colaboró, además, personal docente de las Universidades Juárez Autónoma de Tabasco y Autónoma de Nayarit. Con la colaboración de estas dos instituciones (URSULA Y UMSNH), en 2019 se diseña el Diplomado Internacional Responsabilidad Social Universitaria, Ética y Desarrollo Sostenible: Repensando la labor docente.

En este ejercicio de formación se buscó involucrar a docentes y autoridades universitarias, primeramente, en la reflexión de la participación y corresponsabilidad en la crisis global socio ambiental actual para, posteriormente, destacar el papel que desempeña la educación superior en el reconocimiento, medición, análisis, mitigación y regeneración de los impactos sociales y ambientales 
negativos a través de sus procesos institucionales.

A la fecha, el diplomado se ha ofrecido en dos ocasiones, la primera de noviembre de 2018 a febrero de 2019, con participantes de Argentina, Chile, Colombia, México,
Perú y Uruguay y, debido al gran interés que expresaron personas que no pudieron tomar la primera edición del curso, se abrió una segunda convocatoria para el periodo junio-agosto de 2019, sumándose Ecuador y República Dominicana a la lista de países participantes (Tabla 1).

Tabla 1

Participantes en las dos ediciones del diplomado, por institución de origen y país

\begin{tabular}{|c|c|c|c|}
\hline País/institución & Participantes & País/institución & Participantes \\
\hline Argentina & 2 & Universidad de Guadalajara & 3 \\
\hline Universidad Siglo 21 & 1 & Universidad de Quintana Roo & 8 \\
\hline $\begin{array}{l}\text { Universidad Tecnológica Nacio- } \\
\text { nal }\end{array}$ & 1 & $\begin{array}{l}\text { Universidad Juárez Autónoma } \\
\text { de Tabasco }\end{array}$ & 4 \\
\hline Chile & 12 & $\begin{array}{l}\text { Universidad Juárez del Estado de } \\
\text { Durango }\end{array}$ & 1 \\
\hline Universidad Austral de Chile & 2 & $\begin{array}{l}\text { Universidad Michoacana de San } \\
\text { Nicolás de Hidalgo }\end{array}$ & 17 \\
\hline Universidad de Chile & 1 & $\begin{array}{l}\text { Universidad Nacional Autóno- } \\
\text { ma de México }\end{array}$ & 2 \\
\hline Universidad de Los Lagos & 1 & $\begin{array}{l}\text { Benemérita Universidad Autó- } \\
\text { noma de Puebla }\end{array}$ & 1 \\
\hline Universidad de Talca & 1 & Perú & 24 \\
\hline Universidad del Pacífico & 1 & Universidad Alas Peruanas & 7 \\
\hline Universidad Santo Tomás & 3 & $\begin{array}{l}\text { Universidad Católica de Santa } \\
\text { María }\end{array}$ & 2 \\
\hline $\begin{array}{l}\text { Universidad Tecnológica de Chi- } \\
\text { le INACAP }\end{array}$ & 3 & Universidad del Pacífico & 1 \\
\hline Colombia & 4 & $\begin{array}{l}\text { Universidad Nacional Agraria de } \\
\text { la Selva }\end{array}$ & 1 \\
\hline Universidad Católica de Oriente & 1 & $\begin{array}{l}\text { Universidad Nacional de San } \\
\text { Agustín }\end{array}$ & 1 \\
\hline Universidad de la Costa y IMED & 1 & $\begin{array}{l}\text { Universidad Nacional Santiago } \\
\text { Antúnez de Mayolo }\end{array}$ & 2 \\
\hline $\begin{array}{l}\text { Universidad Minuto de Dios } \\
\text { UNIMINUTO }\end{array}$ & 2 & $\begin{array}{l}\text { Universidad Peruana de Cien- } \\
\text { cias e Informática }\end{array}$ & 1 \\
\hline Ecuador & 1 & $\begin{array}{l}\text { Universidad Privada Antenor } \\
\text { Orrego }\end{array}$ & 4 \\
\hline Universidad Central del Ecuador & 1 & Universidad Privada de Tacna & 2 \\
\hline México & 48 & $\begin{array}{l}\text { Universidad Privada Juan Mejía } \\
\text { Baca }\end{array}$ & 1 \\
\hline Instituto Tecnológico de Sonora & 3 & $\begin{array}{l}\text { Universidad Nacional Agraria La } \\
\text { Molina }\end{array}$ & 1 \\
\hline $\begin{array}{l}\text { Universidad Autónoma de Que- } \\
\text { rétaro }\end{array}$ & 2 & Consejo Departamental de Lima & 1 \\
\hline $\begin{array}{l}\text { Universidad Autónoma de San } \\
\text { Luis Potosí }\end{array}$ & 3 & República Dominicana & 12 \\
\hline $\begin{array}{l}\text { Universidad Autónoma de Ta- } \\
\text { maulipas }\end{array}$ & 1 & $\begin{array}{l}\text { Universidad Católica del Cibao } \\
\text { (UCATECI) }\end{array}$ & 12 \\
\hline $\begin{array}{l}\text { Universidad Autónoma de Zaca- } \\
\text { tecas }\end{array}$ & 1 & Uruguay & 2 \\
\hline $\begin{array}{l}\text { Universidad de Ciencias y Artes } \\
\text { de Chiapas }\end{array}$ & 1 & Universidad de la República & 2 \\
\hline Universidad de Colima & 1 & & \\
\hline
\end{tabular}

Nota: Elaboración propia con base en los registros de inscripción al Diplomado (ambas convocatorias). 
La convocatoria para participar en el diplomado estuvo dirigida a docentes y autoridades de instituciones de educación superior de la región latinoamericana, y fue difundida a través de las redes sociales tanto de URSULA como de la Universidad Michoacana, logrando en ambas ocasiones que los espacios se agotaran en pocos días.

México y Perú destacaron como los países con la mayor cantidad de registros; las áreas del desempeño de las personas participantes fueron, principalmente, Humanidades y Ciencias Sociales (30\%) y EconómicoAdministrativas (26\%), y el $62 \%$ de quienes tomaron el diplomado fueron mujeres.

Este documento expone un análisis de los resultados obtenidos a partir de la impartición de dos diplomados realizados entre 2018 y 2019, frutos de la colaboración entre URSULA y la Universidad Michoacana, y refleja la mirada de la actividad docente de los participantes, su contribución, retos y perspectivas en favor de la ética, la responsabilidad social y el desarrollo sostenible. El objetivo que siguió la investigación aquí descrita consistió en identificar las propuestas realizadas por los participantes en el diplomado, orientadas a contribuir al desarrollo sostenible desde la labor docente en instituciones de educación superior latinoamericanas.

\section{| Método}

Este estudio se desarrolló desde un enfoque mixto, pues en una primera etapa del proceso de investigación, y mediante un análisis de contenido, se analizaron cualitativamente las contribuciones realizadas por quienes participaron en el Diplomado Internacional Responsabilidad Social Universitaria, Ética y Desarrollo Sostenible: Repensando la labor docente. Posteriormente, en una segunda fase de la investigación, se consideró pertinente llevar a cabo un análisis de frecuencias que permitiera cuantificar las propuestas en torno a temáticas específicas.

Los tópicos incluidos en el primer módulo tuvieron como objetivo propiciar la reflexión acerca de cómo los seres humanos nos hemos vuelto insostenibles; es decir, cómo nuestra manera de habitar y desarrollarnos en el planeta ha provocado impactos negativos que ponen en peligro nuestra supervivencia, constatando, por ejemplo, que la industrialización ha generado un sobrecalentamiento de la tierra que ha desencadenado la desaparición acelerada de numerosas especies animales y vegetales, o bien, que el uso creciente de stocks de materias primas como petróleo y minerales está agotando las fuentes de recursos energéticos naturales.

En este sentido, se hizo especial énfasis en llamar la atención hacia la responsabilidad social que debemos asumir, como co-habitantes de la tierra, para frenar y revertir los daños ocasionados por nuestra propia manera de vivir el día a día. Para ello, se solicitó a los participantes que realizaran el cálculo de su huella ecológica y de esclavitud. La primera, en virtud de ser considerada la principal medida mundial de la demanda de la humanidad sobre la naturaleza, ya que mide cuánta área de la tierra y del agua requiere la población humana para producir el recurso que consume y absorber sus desechos usando la tecnología prevaleciente (Global Footprint Network Advancing the Science of Sustainability, 2018); la segunda como "noción sobre el paradigma de producción actual y [de] cómo se exportan los costos sociales de la producción" (Gallardo, 2015, p.6), a través de los hábitos de consumo diario de los seres humanos.

Con ello, se evidenció la urgente necesidad que existe de transitar hacia un modelo de producción y consumo sostenibles, de tal manera que puedan combatirse las causas del deterioro ambiental y de la desigualdad social en el mundo entero, y se solicitó que los participantes reflexionaran acerca de cómo, desde la labor docente, se puede contribuir a la transformación social que requiere 
el desarrollo sostenible.

Para atender esta tarea, los docentes describieron el perfil profesional que, idealmente y desde su perspectiva, debe tener el egresado de educación superior, de tal forma que se proyecte como agente ético del desarrollo sostenible y, posteriormente, idearon algunas estrategias que podrían desarrollar en asignaturas que imparten, para contribuir a la formación de ese perfil profesional ideal.

La actividad solicitada para evaluación del segundo módulo del diplomado consistió en presentar un proyecto social y/o medioambiental en el marco de las actividades curriculares de un curso dictado desde la perspectiva de la metodología del Aprendizaje y Servicio Solidario. Se dejó abierta la posibilidad de que los docentes presentaran un proyecto que ya hubieran llevado a la práctica o bien, que diseñaran una idea nueva a partir de los conceptos, ejemplos y actividades realizadas en el diplomado.

Es importante mencionar que el enfoque del Aprendizaje y Servicio Solidario fue resaltado durante el diplomado como una pedagogía que permite a la universidad involucrarse en el desarrollo social, y cuyas prácticas se caracterizan por articular la adquisición de conocimientos, la reflexión sobre las condiciones sociales de vida y la transformación de la realidad, y que se trata, por tanto, de sostener simultáneamente dos intencionalidades: la intencionalidad pedagógica de mejorar la calidad de los aprendizajes y la intencionalidad solidaria de ofrecer una respuesta participativa a una necesidad social (Tapia, 2017).

Así mismo, se enfatizó en la diferenciación de esta pedagogía con otras prácticas educativas y solidarias que son realizadas en el contexto universitario. Para lograr una mejor comprensión de dichas diferencias resultó útil hacer referencia a los Cuadrantes del aprendizaje y servicio desarrollados por la Universidad de Standford y adaptados por María Nieves Tapia:
- Trabajos de campo/pasantías/aprendizaje en base a problemas: en este cuadrante agrupamos las actividades de investigación y práctica que involucran a los estudiantes con la realidad de su comunidad, pero considerada como objeto de estudio [...] Los destinatarios de la actividad son los mismos estudiantes, el énfasis está puesto en la adquisición de aprendizajes, y el contacto con la realidad comunitaria es exclusivamente instrumental.

- Actividades solidarias asistemáticas: se definen por su intencionalidad solidaria y por la falta de articulación con el aprendizaje formal. [..]. Son "asistemáticas" porque surgen a partir de situaciones fortuitas [...] atienden una necesidad específica en un lapso acotado y no son planificadas institucionalmente. El principal destinatario del proyecto es la comunidad beneficiaria [...]. El énfasis está puesto en atender una necesidad, y no en generar una experiencia educativa.

- Actividades solidarias institucionales: actividades de carácter institucional y

sistemático orientadas a promover actividades solidarias, de compromiso social y participación ciudadana para los jóvenes como una expresión de la misión institucional. [...].

- Aprendizaje-servicio solidario: [...] ubicamos las experiencias, prácticas y programas que ofrecen simultáneamente una alta calidad de servicio y un alto grado de integración con los aprendizajes curriculares [...]. El énfasis está puesto tanto en la adquisición de aprendizajes como en el mejoramiento de vida de una comunidad concreta (Tapia, 2017, pp. 15-16).

Durante el tercer módulo se enfatizó en la necesidad de revalorar a las universidades como agentes del cambio 
social y, particularmente, en la urgencia de que estas instituciones se involucren directamente en el logro de los objetivos y metas contenidas en la agenda 2030 para el desarrollo sostenible, colaborando con el resto de actores que coexisten tanto en el ámbito público como en el privado y en sectores ajenos a la academia.

En este contexto, la tercera actividad para evaluación consistió en describir actividades, contenidos o proyectos con los que se pueda incidir para cumplir las metas contenidas en los objetivos de desarrollo sostenible (ODS), según la perspectiva de los docentes.

Por último, ya que los participantes se habían apropiado del concepto de RSU y habían reflexionado sobre el papel tan importante que tienen como gestores institucionales e integrantes de la comunidad académica en instituciones de educación superior, se les solicitó que voltearan la mirada al interior de sus instituciones con la intención de identificar qué prácticas universitarias pudieran estar contribuyendo al logro de los ODS y que, con dicha información, realizaran una especie de mapeo en cada uno de los ámbitos de la RSU.

Para determinar las categorías de análisis, se tomó como referencia el Modelo URSULA de RSU (Figura 1), en virtud de que constituye un marco general que integra las discusiones teóricas más recientes acerca de RSU y concibe a ésta en su carácter transversal, que permea los cuatros procesos institucionales de gestión organizacional, formación, cognición y participación social.

\section{Figura 1}

Ámbitos y metas que integran el Modelo URSULA de RSU

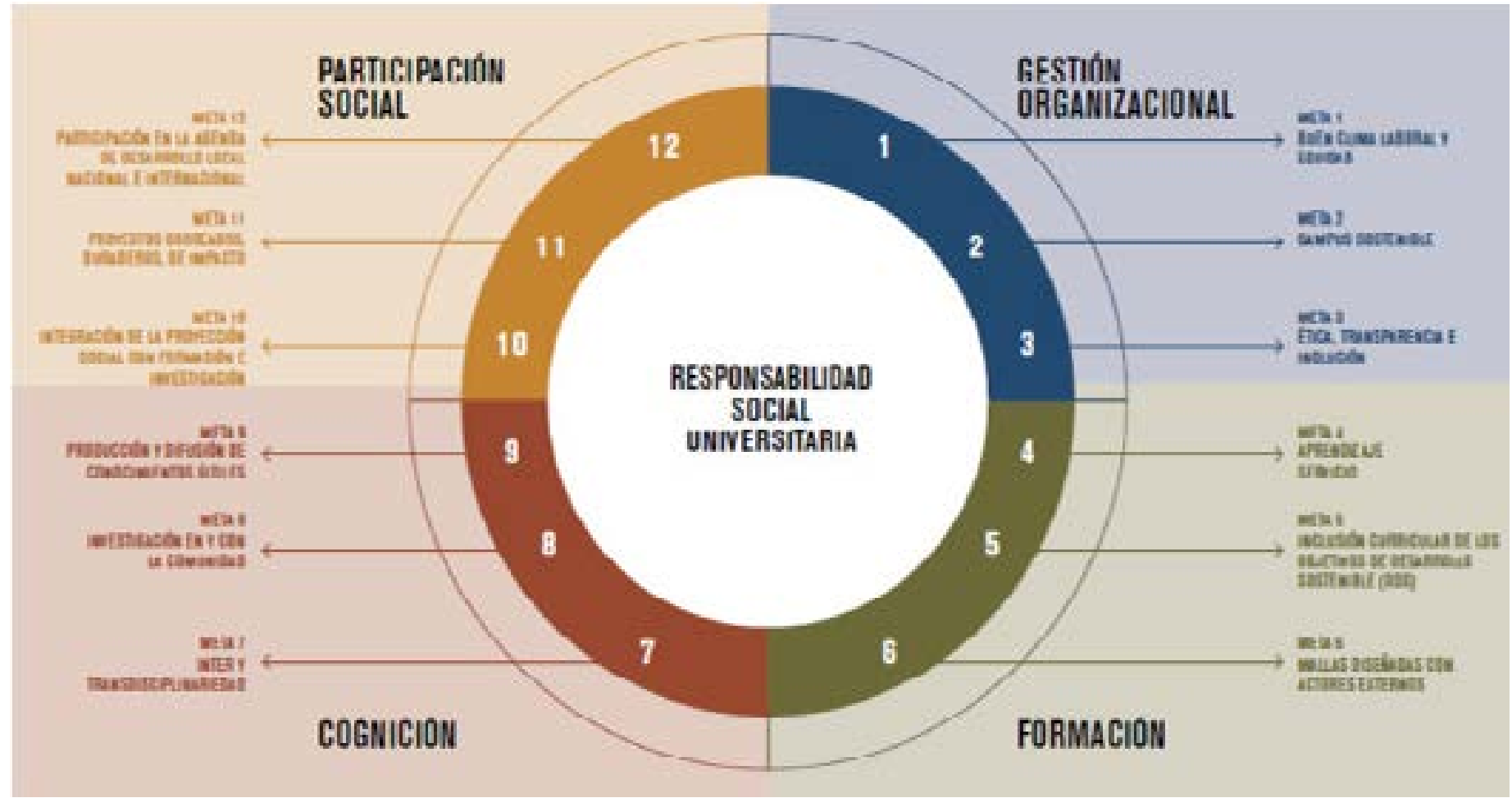

Nota: Vallaeys (2020b, p. 62). 
Considerando estos ámbitos y metas, se revisaron 410 contribuciones docentes. Para organizar y sistematizar la información, se construyó una plantilla en Excel en la que se clasificaron las propuestas de acuerdo a los siguientes criterios: año de edición del diplomado, país de origen de la IES de adscripción del participante, área del conocimiento en la que desempeña su labor docente, título de la propuesta específica de acuerdo a la tarea solicitada, ámbitos de acción y metas del Modelo URSULA.

Una vez capturada la información en dicha plantilla, a partir del análisis de contenido, se procedió a realizar el análisis de frecuencias y, a partir de ellas, definir la participación relativa que las propuestas tienen en cada uno de los ámbitos: gestión organizacional, formación, cognición y participación social, así como en sus respectivas metas.

\section{| Resultados}

La mayoría de las reflexiones en torno a estrategias docentes para contribuir a la formación de egresados universitarios comprometidos con el desarrollo sostenible se concentraron en el ámbito de la formación (58\% del total de las propuestas analizadas), atendiendo principalmente a la necesidad de incluir los ODS en el currículo universitario (ver figura 2).

\section{Figura 2}

Clasificación de las estrategias docentes para contribuir a la formación de egresados universitarios comprometidos con el desarrollo sostenible, según ámbito de acción y metas de la RSU

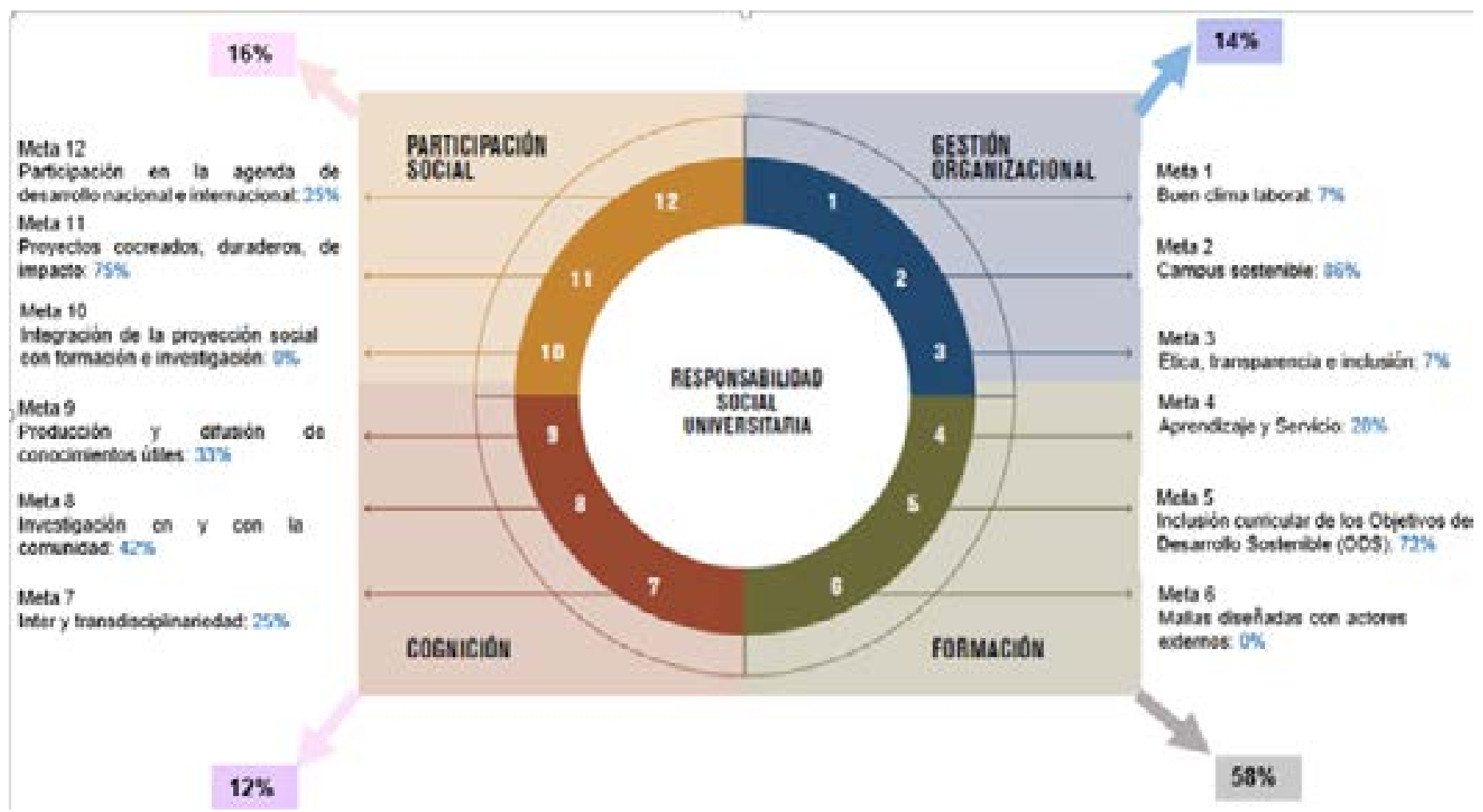

Nota: Adaptación propia con base en el Modelo URSULA de RSU. 
Entre las estrategias más destacadas por los docentes se encuentran: la identificación de la necesidad de desarrollar nuevas actitudes desde la docencia en el trabajo con jóvenes, la incorporación de criterios de sostenibilidad en los programas de sus asignaturas, el desarrollo de guías de aprendizaje y metodologías de enseñanza con el enfoque del desarrollo sostenible; impartición de talleres sobre derechos humanos y sostenibilidad, promoción del involucramiento de los estudiantes en la realización de campañas de concientización ambiental dirigidas a la población en general, fomento del debate y el consenso sobre los temas del desarrollo sostenible y, en particular, sobre cómo contribuir al cumplimiento de los ODS; cultivar valores y enseñar al estudiantado a comprender los conflictos ambientales y poder resolverlos a través de la aplicación in situ de la teoría que se les brinda.

En cuanto a los proyectos de aprendizaje y servicio solidario pensados desde el ejercicio de la docencia se identificó que, aun cuando en su definición se establece la doble intencionalidad de la formación y la solidaridad, los docentes privilegian el aspecto formativo dejando de lado la participación social.

Algunos proyectos que lograron integrar ambas intenciones fueron: Crianza de cuyes y desarrollo social sostenible (Perú), Centro Comunitario Comunidad de Dios (Perú), Clubes de adultos mayores (Chile), Desarrollo integral de la comunidad indígena Tepehuana (México), Asesorías financieras a comedores populares (Uruguay), por mencionar algunos.

En relación a la dimensión de la Gestión Organizacional se registraron pocos proyectos, la totalidad de los cuales correspondían a proyectos enfocados a la meta Campus sostenible y a la gestión de desechos sólidos, quedando sin participación la meta Buen clima laboral y Ética, transparencia e inclusión (figura 3).

Figura 3

Clasificación de Proyectos de Aprendizaje y Servicio Solidario, según ámbito de acción y metas de la RSU

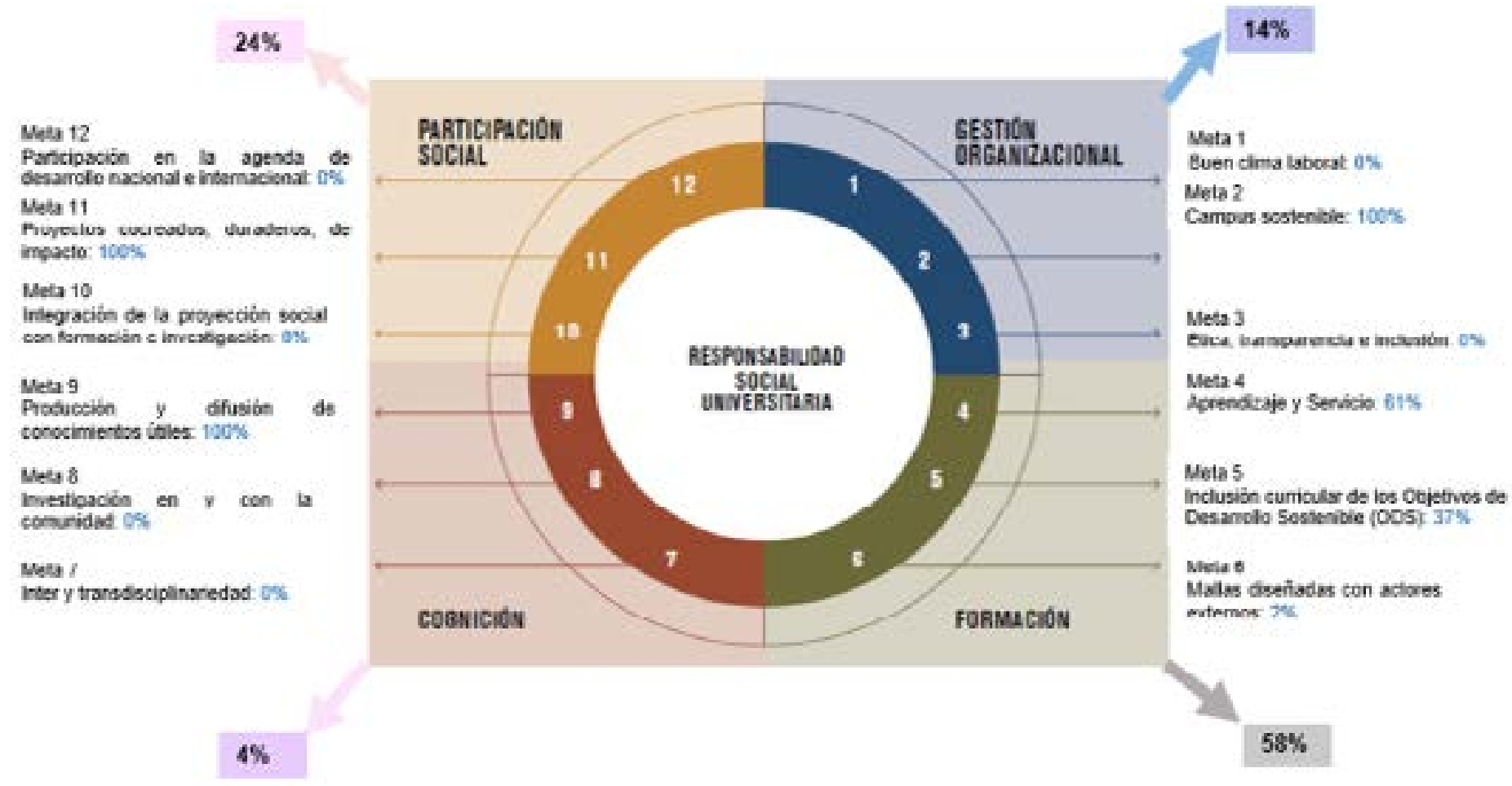

Nota: Adaptación propia con base en el Modelo URSULA de RSU. 
De los proyectos ubicados en la dimensión de Formación, la mayoría se encuentra en la meta de Aprendizaje y Servicio, sin embargo, se observa que los ODS empiezan a ser parte de los proyectos en las universidades. Palabras que duelen: género, migración y violencia, (México); Reforestación con plantas nativas de suelos degradados por tala ilegal en la selva de Ucayali (Perú), y Programa de reforestación de la loma de Guaigui (República Dominicana), son algunos ejemplos.

La dimensión de la Cognición es muy poco visible en este tipo de proyectos y las propuestas se centraron en la Producción y difusión de conocimientos útiles. Algunas de éstas son: Observatorio de medios sobre la calidad del sistema educativo nacional (República Dominicana), El nopal, una estrategia para la mejora de productos lácteos en zonas rurales (México), y Monitoreo y análisis de la calidad de las aguas (México).

En las propuestas para contribuir a los Objetivos de Desarrollo Sostenible desde la labor docente puede observarse que éstas se concentran en la Inclusión curricular de los ODS (61\%, meta 5), aunque su presencia no siempre es explícita, la aportación es visible. Otro aspecto significativo es que el Aprendizaje y Servicio Solidario es utilizado como herramienta pedagógica para alcanzar esta meta (figura 4).

\section{Figura 4}

Clasificación de las propuestas para contribuir a los Objetivos de Desarrollo Sostenible desde la labor docente, según ámbito de acción y metas de la RSU

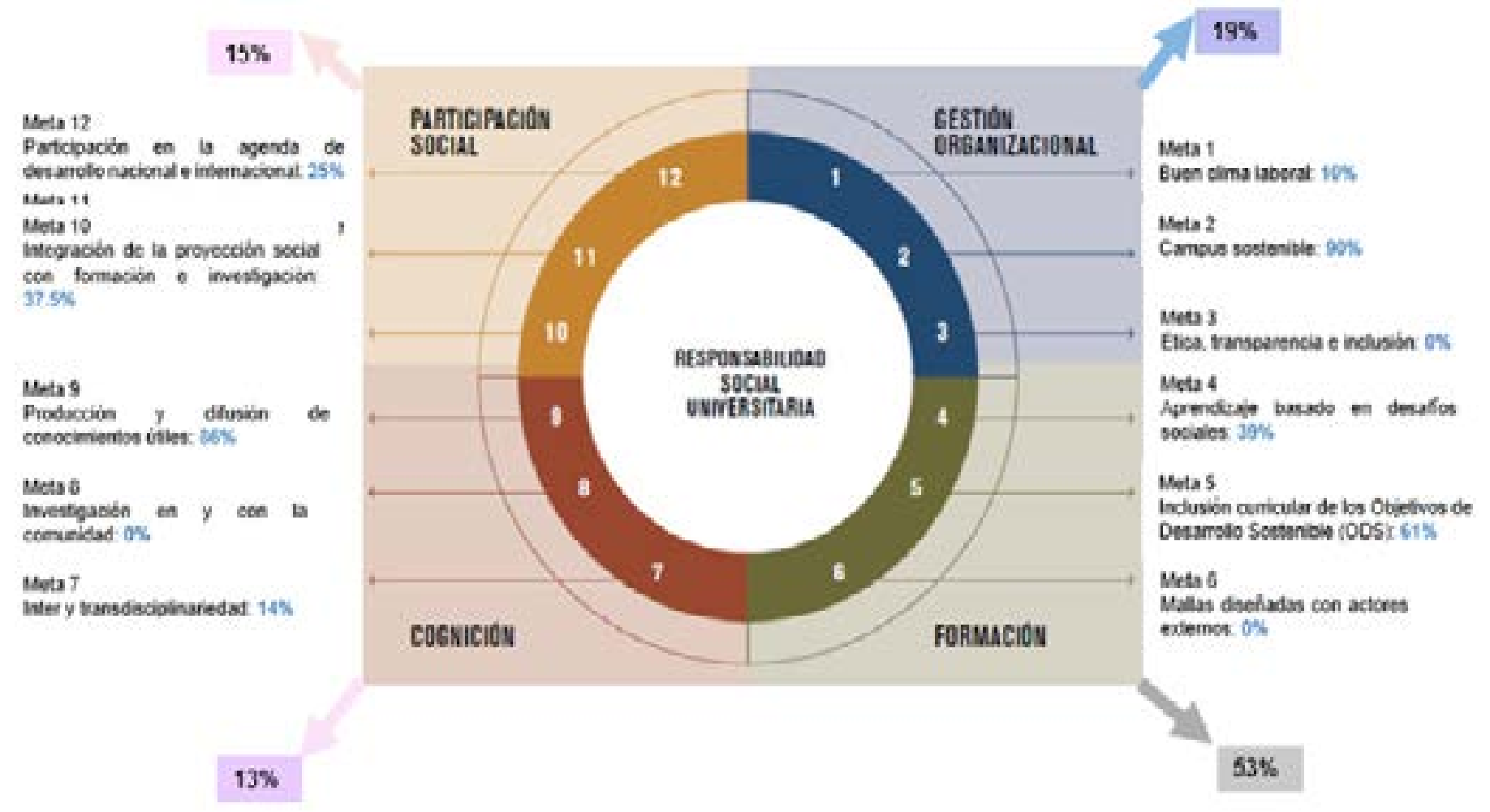

Nota: Adaptación propia con base en el Modelo URSULA de RSU. 
Algunos ejemplos de aportaciones en este sentido son: Capacitación a través de los Embajadores de los ODS (Perú), Feria por los ODS (México), Asignatura de Innovación Social (Chile), Práctica pre-profesional con perspectiva de Responsabilidad Social en el marco de los ODS (Argentina) y Pasantía en un centro de rehabilitación (Uruguay).

Nuevamente, la mayoría de las propuestas dentro de la dimensión de la Gestión Organizacional se encuentran en la meta Campus sostenible, con poca incidencia en el Buen clima laboral y Ética, transparencia e inclusión. Pueden citarse, como ejemplos, los siguientes: La Universidad y los ODS (Perú), Autodiagnóstico de RSU (Colombia), Creación de red de tenderos emprendedores sostenibles proveedores de servicios de alimentación para universidades y empresas del barrio Minuto de Dios (Colombia).

Por lo que se refiere al ámbito de la Cognición, se aprecia que hay interés de los participantes en la Producción y difusión de conocimientos útiles que contribuyan a los ODS, sin embargo, suscribir proyectos inter y transdisciplinarios tiene menor interés y la Investigación en y con la comunidad se queda totalmente desatendida.
En cuanto a la Participación Social existe una distribución muy equitativa en las tres metas del Modelo URSULA: Integración de la proyección social con la formación e investigación, Proyectos cocreados, duraderos y de impacto y Participación en la agenda de desarrollo nacional e internacional.

Finalmente, en la revisión de los proyectos que los docentes identificaron como buenas prácticas para contribuir a los Objetivos de Desarrollo Sostenible implementadas en sus instituciones de origen, se encontró que la mayoría de ellas corresponden al ámbito de la gestión organizacional (39\%), particularmente orientadas a lograr un campus sostenible (ver figura 5). Sin embargo, se evidencia una visión muy acotada al tema del cuidado del medio ambiente y sólo se tocan de forma marginal aspectos relacionados con lograr un ambiente laboral armónico, en el que se establezcan relaciones de igualdad entre los géneros, las razas, las culturas y las capacidades y en donde se privilegie el desarrollo creativo, colaborativo y sinérgico de todos los miembros de la comunidad universitaria, así como la ética, la transparencia y la rendición de cuentas.

\section{Figura 5}

Clasificación de las buenas prácticas universitarias para contribuir a los Objetivos de Desarrollo Sostenible, según ámbito de acción y metas de la RSU

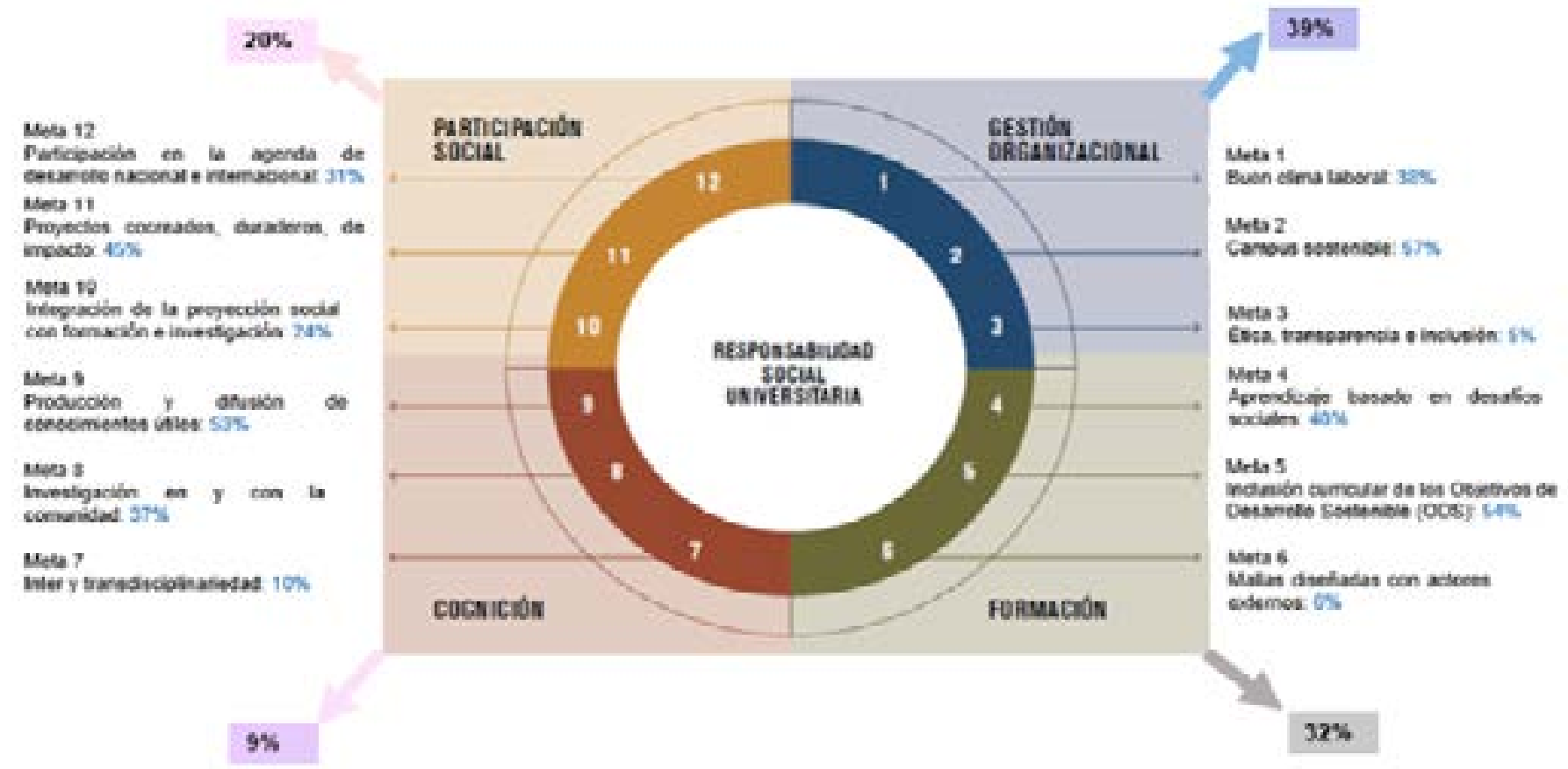

Nota: Adaptacion propia con base en el Modelo URSULA de RSU. 


\section{| Discusión}

Al asumir que la labor del docente universitario va más allá de proporcionar conocimientos técnicos que permitan el desenvolvimiento profesional del egresado de educación superior, y reconocer que puede llegar a incidir en la adquisición de aprendizajes y el desarrollo de competencias para la vida (Vera, et al., 2012), pone en evidencia, a su vez, la responsabilidad social que tienen las universidades, así como el compromiso que deben asumir para garantizar que la formación que ofrecen sea de calidadad y socialmente pertinente. Valleys (2021, p. 52) señala al respecto: "no hay calidad de la educación sin pertinencia social de la educación, sustentada en una sólida política de gestión socialmente responsable de las IES".

Las propuestas realizadas por los docentes universitarios lationamericanos que participaron en el Diplomado Internacional Responsabilidad Social Universitaria, Ética y Desarrollo Sostenible: Repensando la labor docente, dan cuenta de que fue posible una reflexión importante en torno a la necesidad de gestionar de forma socialmente responsable la formación académica. En este sentido, puede considerarse un avance significativo en términos de construir el camino que nos permita llegar al objetivo de que "el aula de clase se constituya en el escenario para la puesta en marcha del proyecto educativo, que active el desarrollo axiológico a partir de la interacción entre estudiantes y docentes" (González \& González, p. 100).

Sin embargo, es importante mencionar que aun es necesario reforzar la reflexión acerca de los otros ámbitos de acción de la RSU, principalmente en torno al de la cognición, ya que muy pocas propuestas reflejan un cuestionamiento acerca de los modelos epistemológicos que se promueven desde el aula, y no se identificó ninguna propuesta en términos del diseño de mallas en colaboración con actores externos y de la integración de la proyección social con formación e investigación, dentro de los ámbitos de formación y participación social, respectivamente. Puede señalarse, por tanto, que aún persiste una cultura universitaria que la caracteriza como una torre de marfil (Shapin, 2012), poco interesada y nada dispuesta a salir de su propio claustro y a permitir el vínculo con la sociedad, no sólo de adentro hacia afuera, sino de afuera hacia adentro. Es necesario avanzar

Para superar esta situación, es necesario fomentar vínculos con otros actores sociales del "entorno local, regional y nacional [y poner] particular atención a los cambios de orden global que han transformado el campo universitario hacia una cultura de innovación y [responsabilidad]" (González \& Schneider, 2020, p. 26).

Otro hallazgo relevante es que, dentro de las propuestas analizadas, se encuentra la tendencia a considerar los medios como fines, como en el caso de los procesos de capacitación, los cuales son considerados contribuciones a los ODS. Sin embargo, no se lleva a cabo un seguimiento del impacto que se logra con los cursos y talleres y, por otra parte, los indicadores de evaluación se centran en el número de cursos y/o de participantes. En este sentido, es necesario enfatizar en la formación docente y dejar claro que la RSU no es una acción y que, como encargados de su gestión en las aulas, los docentes deben evitar prácticas que los conviertan en administradores de "herramientas de RSU, [encargados de llenar] listas de indicadores ad hoc para el reporte anual de sostenibilidad, que nadie leerá y que no cambiará (casi) nada" (Valleys, 2021, p. 28).

Así mismo, resulta sumamente importante insistir en que es momento de repensar la labor docente desde su aporte o contribución a los ODS, y generar la necesidad del enfoque integral, inter, multi y transdisciplinario, con participación real de los involucrados, en una realidad específica y con estrecha vinculación entre el saber académico y el saber popular. 
Finalmente, otro hallazgo relevante tiene que ver con la escasa evidencia encontrada por los participantes en cuanto al ámbito de la cognición, pues puede entenderse como la falta de cuestionamiento desde las mismas universidades acerca del tipo de conocimientos que produce, así como las formas y medios a través de los cuales los difunde y transmite a la sociedad para garantizar que ésta se apropie de ellos para la solución de los problemas que le aquejan. En particular, un aspecto que denota una debilidad importante tiene que ver con la monodisciplinariedad que aún persiste en la comunidad académica universitaria, y que debe ser modificada para transitar hacia un abordaje de la realidad social en la que dialoguen las distintas áreas del saber científico y de otros saberes no especializados, pues "la transdisciplinariedad establece un nuevo modo de relación con el saber; y por tanto, se convierte en una categoría clave para cualquier reforma o cambio educativo" (Pérez, et al., 2013, p. 18).

\section{| Conclusiones}

Uno de los aspectos más importantes que se ha podido estudiar en este análisis exploratorio es la importancia de la participación docente en los procesos de la adopción de la RSU como enfoque de gestión, ya que es a través de su mediación que los estudiantes se acercan al conocimiento, lo interpretan y aplican a la solución de problemas de la realidad. Por esta razón, la labor docente debe ser revalorada, pues es a través del ejercicio de esta vocación que se materializa la pertinencia social de la formación profesional mediante el desarrollo de prácticas y contenidos que acerquen a los estudiantes a la realidad económica, social y ambiental en que viven.

Por otra parte, debe reconocerse la necesidad de superar ciertos espejismos de la visión tradicional de la enseñanza-aprendizaje, ya que aún se observan proyectos que conservan, como se ha dicho, la postura de la torre de marfil, que considera que en la Universidad se tiene el conocimiento y la solución a todos los problemas sin aceptar que cada vez más el conocimiento se encuentra en todos lados, para de esta manera alcanzar las metas de proyectos cocreados, duraderos y de impacto, la investigación en y con la comunidad, además de la inter y transdisciplinariedad.

Con este nuevo enfoque, en el que la RSU, la ética y el desarrollo sostenible sean criterios transversales que estén presentes en todos los proyectos ya sean de gestión, formación, investigación o participación social, se logrará construir propuestas que lleven a contribuir a los ODS, cumpliendo así con el rol transformador esencial que tienen las instituciones de educación superior.

En la otra cara de la moneda tenemos a los estudiantes, quienes deberán asumir el protagonismo que les corresponde, y los docentes tendrán que aprender a confiar en sus capacidades, permitiéndoles ser los y las líderes que conduzcan los proyectos, para que así se cumpla con el objetivo formativo de las tareas. En este sentido, hay que señalar lo necesario que es que estudiantes y docentes dejen el aula y entren en contacto con las problemáticas del territorio en el que se desarrollan

\section{| Referencias}

Gallardo, M. (21 de marzo de 2015). Herramienta que permite calcular cuántos "esclavos" trabajan para ti sin que lo sepas. https://cutt.ly/6TCBjWx

Global Alliance of Tertiary and Higher Education Sustainability and Student Networks [EAUC \& ACTS]. (2021). Annual SDG Accord Report 2021. Progress towards the Global Goals in the University and College sector. https:// cutt.ly/KTCB8ec

González, E. \& González, M. (2012). La educación para la responsabilidad social. Una mirada desde el docente universitario. Interacción y Perspectiva. Revista de Trabajo Social, 2(2), 97-106. https://cutt.ly/TTCBTtJ

González, G. \& Schneider, C. (2020). Más allá de la Torre de Marfil: vinculaciones de la universidad y sus te- 
rritorios. Revista Iberoamericana de Educación Superior, XI(31), 24-47. https://doi.org/h9fv

Pérez, E., Moya, A. \& Curcu, A. (2013). Transdisciplinariedad y educación. Educere, 17(56),15-26. https://cutt. ly/gTCBDWp

Ramos Torres, D. I. R. (2021). Contribution of higher education to the Sustainable Development Goals from teaching. Revista Española de Educación Comparada, 37(37), 89-110. https://doi.org/h9fw

Shapin, S. (2012). The ivory tower: The history of a figure of speech and its cultural uses. BJHS, 45(1), 1-27. https:// doi.org/gfb286

Tapia, M. (2017). Cómo desarrollar proyectos de aprendizaje y servicio solidario en educación media: secundaria y enseñanza técnica. Centro Latinoamericano de Aprendizaje y Servicio Solidario. https://cutt.ly/gTCNflh

Unión de Responsabilidad Social Universitaria Latinoamericana [URSULA] (2020). Quienes Somos | URSULA. https://cutt.ly/DTCNjTC

Vallaeys, F. (2020a). Hacia una política pública latinoamericana de Responsabilidad Social Universitaria: Innovación social, calidad y pertinencia de la educación superior. Editorial CAF/URSULA. https://cutt.ly/xTCNNms

Vallaeys, F. (2020b). El Modelo URSULA. Estrategias, herramientas e indicadores. Unión de Responsabilidad Social Universitaria Latinoamericana. https://cutt.ly/ vTCN7VD

Vallaeys, F., \& Álvarez Rodríguez, J. (2019). Hacia una definición latinoamericana de Responsabilidad Social Universitaria. Aproximación a las preferencias conceptuales de los universitarios. Educación XX1, 22(1), 93-114. https://doi.org/10.5944/educXX1.19442

Vera, L., Gómez, M., Coromoto Y. \& Perozo, L. (2012). La docencia en el marco de la responsabilidad social universitaria. Opción,1(68), 257-172. https://cutt.ly/ CTCMx63 